\title{
REPRESENTATION OF PSEUDO-LATTICE ORDERED VECTOR SPACES
}

\author{
RICHARD C. METZLER
}

(Received 18 February 1977)

Communicated by A. P. Robertson

\begin{abstract}
Ordered vector spaces more general than vector lattices are represented as function spaces with 'almost' the pointwise order. The 'pseudo-lattice' property which is necessary (and sufficient in the finite-dimensional case) for the representation is also studied in relation to the lattice property.
\end{abstract}

Subject classification (Amer. Math. Soc. (MOS) 1970): 06 A 65.

Representation of an ordered vector space as a space of functions works well when the space is an Archimedean lattice. Also if the space is 'locally finite dimensional' and has the Riesz decomposition property we have shown in Metzler (1969) that it can be represented as a 'selected order space' of functions. The representations of spaces with the decomposition property form a proper subclass of the selected order spaces and in this paper we investigate the problem of characterizing those spaces having selected order space representations. The order property characterizing these spaces is given here and spaces having this property are christened pseudo-lattices. Geometrically, in finite dimensions, these spaces correspond to those with positive cones such that if any interior element of a face is positive then the whole interior of the face is positive. We also show that if we take a natural 'closure' of the positive cone of a pseudo-lattice we obtain the positive cone of a lattice.

Let $V$ be a directed ordered vector space with positive cone $V^{+}$. (Notation is that of Metzler (1975) except that we assume that $V^{+} \cap\left(-V^{+}\right)=\{0\}$ unlike the assumption there.) We say $V$ is a pseudo-lattice if, for every pair $(x, y) \in V^{+} \times V^{+}$, there exists a positive element $z$ such that: (i) for all $p \in V, p \geqslant\{x, y\}$ implies $p+\alpha x \geqslant z$ 
for all $\alpha>0$ (the least property); and (ii) $z+\alpha x \geqslant\{x, y\}$ for all $\alpha>0$ (the upper property). Clearly if $\alpha$ were allowed to be zero then $z$ would just be the usual least upper bound.

For any positive element $x$, we define $\left(V^{+}\right)_{x}=\bigcap_{\alpha>0}\left(-\alpha x+V^{+}\right)$and let $V^{+\sim}=\bigcup_{x \in V^{+}}\left(V^{+}\right)_{x}$. It can be shown easily that $V$ is Archimedean if and only if $V^{+}=V^{+} \sim$ (Metzler (1969), p. 3). It is also clear that $V$ is almost Archimedean if and only if $\left(V^{+}\right)_{x} \cap-\left(V^{+}\right)_{x}=\{0\}$ for all $x \in V^{+} \mid\{0\}$.

THEOREM 1. If $V$ is an almost Archimedean pseudo-lattice then $\left(V, V^{+\sim}\right)$ is an Archimedean lattice and there is exactly one $z$ having the least and upper properties for each $(x, y)$; it is $x \vee y$ (sup taken in the $V^{+\sim}$ order).

Proof. Since $V$ is directed, to show that $V$ with the $V^{+\sim}$ order is a lattice, it is sufficient to show any two elements of $V^{+}$have a least upper bound. Let $x, y$ and $z$ be as in the definition of pseudo-lattice. We will show that $z=x \vee y$ by showing that $z+V^{+\sim}=\left(x+V^{+\sim}\right) \cap\left(y+V^{+\sim}\right)$. Suppose $b \in z+V^{+\sim}$. Then there is $d \in V^{+}$ such that $b+\alpha d \in z+V^{+}$for all $\alpha>0$ and this shows that

$$
\{b-x+\alpha(d+x), b-y+\alpha(d+x)\} \subset V^{+}
$$

for all $\alpha>0$. Thus $b-x \in\left(V^{+}\right)_{d+x}$ and $b-y \in\left(V^{+}\right)_{d+x}$ and so

$$
b \in\left(x+V^{+\sim}\right) \cap\left(y+V^{+\sim}\right) .
$$

This gives the inclusion $z+V^{+\sim} \subset\left(x+V^{+\sim}\right) \cap\left(y+V^{+\sim}\right)$.

Now suppose that $a \in\left(x+V^{+\sim}\right) \cap\left(y+V^{+\sim}\right)$. Then there are elements $m$ and $n$ in $V^{+\sim}$ such that $a=x+m=y+n$. By definition of $V^{+\sim}$ there are $p_{1}, p_{2}$ in $V^{+}$ such that $m+\alpha p_{1} \in V^{+}$and $n+\alpha p_{2} \in V^{+}$for all $\alpha>0$. If we let $p=p_{1}+p_{2}$ we have $\{m, n\}+\alpha p \subset V^{+}$for all $\alpha>0$. Therefore $a+\alpha p=x+m+\alpha p=y+n+\alpha p$ and we see that $a+\alpha p \in\left(x+V^{+}\right) \cap\left(y+V^{+}\right)$for all $\alpha>0$. Hence $a+\alpha p+\alpha x \in z+V^{+}$for all $\alpha>0$ by the least property. This means $a-z \in\left(V^{+}\right)_{p+x}$ so $a \in z+V^{+} \sim$. We conclude $\left(x+V^{+\sim}\right) \cap\left(y+V^{+\sim}\right) \subset z+V^{+\sim}$.

Now if $z^{\prime}$ also has the least and upper properties with respect to $x$ and $y$ then $z^{\prime}+V^{+\sim}=\left(x+V^{+\sim}\right) \cap\left(y+V^{+\sim}\right)=z+V^{+\sim}$. Therefore $\left\{z-z^{\prime}, z^{\prime}-z\right\} \subset V^{+\sim}$ which means there exists $q \in V^{+}$such that $\left\{z-z^{\prime}, z^{\prime}-z\right\} \subset\left(V^{+}\right)_{q}$. We conclude $z=z^{\prime}$ since $V$ is almost Archimedean.

To see that $V$ is Archimedean in the $V^{+\sim}$ order, suppose that $y \in V^{+\sim}$ and $x \in n^{-1} y-V^{+\sim}$ for all $n=1,2, \ldots$. Choose $z \in V^{+}$such that $y+\alpha z \in V^{+}$for all $\alpha>0$ and such that $y-x+\frac{1}{2} z \in V^{+}$(since $V$ is directed such a $z$ can be found). Then $n^{-1} y-x+(y+z)=(y-x)+\frac{1}{2} z+n^{-1}\left(y+\frac{1}{2} n z\right) \in V^{+}$for all $n$. Now

$$
(y+z) \vee\left(n^{-1} y-x+y+z\right)=n^{-1} y-x+y+z
$$

since $n^{-1} y-x \in V^{+\sim}$ and the left side is the least upper bound in the $V^{+\sim}$ order. 
Noting that $y+z$ and $n^{-1} y-x+y+z$ are both in $V^{+}$we can apply the upper property in the following equality:

$$
\begin{aligned}
-x+n^{-1}(2 y+z) & =n^{-1} y-x+n^{-1}(y+z)=n^{-1} y-x+y+z+\left(n^{-1}-1\right)(y+z) \\
& =\left[(y+z) \vee\left(n^{-1} y-x+y+z\right)\right]-(y+z)+n^{-1}(y+z) \\
& \in V^{+}
\end{aligned}
$$

for all $n$. Thus we conclude that $-x \in V^{+\sim}$ which means that $V^{+\sim}$ is Archimedean.

We note that this theorem shows that the asymmetry in the definition of pseudolattice $(z+\alpha x$ rather than $z+\alpha y)$ is only apparent in the almost Archimedean case.

THEOREM 2. If $V$ is a pseudo-lattice such that the element $z$ of the definition is unique for every pair of positive elements then $V$ is almost Archimedean.

Proof. Suppose $-\alpha b \leqslant a \leqslant \alpha b$ for $b \in V^{+}$and $\alpha>0$. We must show $a=0$ to conclude that $V$ is almost Archimedean. We note first the easily proved fact that these inequalities are equivalent to the statement $r a+b \geqslant 0$ for all real $r$.

We will show that $a+b$ and $-a+b$ both have the least and upper properties with respect to the pair $(a+b,-a+b)$ and uniqueness then gives $a+b=-a+b$ yielding $a=0$.

Clearly $a+b$ and $-a+b$ have the least property with respect to $(a+b,-a+b)$ and we need only show each has the upper property. We note that $a+b \geqslant a+b$, while $a+b-(-a+b)+\alpha(a+b)=\alpha(((2 / \alpha)+1) a+b) \in V^{+}$. Similarly, $-a+b \geqslant-a+b$, while $(-a+b)-(a+b)+\alpha(a+b)=\alpha((1-(2 / \alpha)) a+b) \in V^{+}$.

Changing the definition in Metzler (1969) slightly we define a selected order space $W$ to be a directed ordered linear space of functions on a set $M$ such that $W^{+}$is contained in the cone of nonnegative functions and such that any nonnegative function in $W$ with the same support as a function in $W^{+}$is also in $W^{+}$. (Note: support $f=\operatorname{spt} f=\{m \in M: f(m) \neq 0\}$.) A geometric example of this is given by the case $M=\{1,2,3\}$. Then $W^{+}$would be the interior of the positive octant in $\mathbf{R}^{3}$ together with the origin and some or all of the edges and some or all of the faces. The 'support' property above ensures that if any interior element of a face is in $W^{+}$then the entire interior is in $W^{+}$.

THEOREM 3. Every selected order space $V$ with the property that the $V^{+\sim}$ ordering is a lattice ordering is an almost Archimedean pseudo-lattice.

Proof. We show first that, if $V$ is a selected order space, then $V^{+\sim}$ is the cone of nonnegative functions in $V$. Given $f \in V^{+\sim}$ there exists $g \in V^{+}$such that $f+\alpha g \in V^{+}$for all $\alpha>0$. Thus $(f+\alpha g)(m) \geqslant 0$ for all $\alpha>0$ which shows $f$ is nonnegative. Conversely if $h \in V$ is non-negative then, since $V$ is directed, there exist 
$p$ and $q$ in $V^{+}$such that $h=p-q$. It is easy to see that $\operatorname{spt}(h+\alpha p)=\operatorname{spt} p$ for all $\alpha>0$ so we have $h+\alpha p \in V^{+}$which gives $h \in V^{+}$. We note that the almost Archimedean property follows from the fact that $V^{+\sim}$ is the cone of nonnegative functions in $V$.

Given $x$ and $y$ in $V^{+}$, we will show that $x \vee y$, taken in the $V^{+\sim}$ (pointwise) order, has the least and upper properties with respect to $x$ and $y$. Considering the least property first, if $a \in\left(x+V^{+}\right) \cap\left(y+V^{+}\right)$, then let

$$
h=a-(x \vee y)+\alpha x=(a-x+\alpha x) \wedge(a-y+\alpha x)
$$

for $\alpha>0$. Then $\operatorname{spt} h=\operatorname{spt}(a-x+\alpha x) \cap \operatorname{spt}(a-y+\alpha x)=\operatorname{spt}(a) \cap \operatorname{spt}(a-y+\alpha x)$. (Note that $a(m)>0$ if and only if $a(m)-x(m)+\alpha x(m)>0$ since $a-x \in V^{+}$.) Now the last term equals

$$
\begin{aligned}
\operatorname{spt} a \cap[\operatorname{spt}(a-y) \cup \operatorname{spt}(\alpha x)] & =[\operatorname{spt} a \cap \operatorname{spt}(a-y)] \cup[\operatorname{spt} a \cap \operatorname{spt} \alpha x] \\
& =\operatorname{spt}(a-y) \cup \operatorname{spt}(\alpha x)=\operatorname{spt}(a-y+\alpha x) .
\end{aligned}
$$

Since $a-y+\alpha x \in V^{+}$we see that $h \in V^{+}$. Thus $x \vee y$ has the least property.

For the upper property let $g=x \vee y+\alpha x-x$. Then $g=[0 \vee(y-x)]+\alpha x$ and

$$
\begin{aligned}
\operatorname{spt} g & =\operatorname{spt}[0 \vee(y-x)] \cup \operatorname{spt}(\alpha x)=\{m \in M: y(m)>x(m)\} \cup\{m \in M: x(m)>0\} \\
& =\operatorname{spt}(x+y) ;
\end{aligned}
$$

so $g \in V^{+}$. Finally, if $f=x \vee y+\alpha x-y$ then $f=(x-y) \vee 0+\alpha x$ and

$$
\text { spt } f=[\operatorname{spt}((x-y) \vee 0)] \cup \operatorname{spt}(\alpha x)=\{m: x(m)>y(m)\} \cup\{m: x(m)>0\}=\operatorname{spt} x
$$

and so $f \in V^{+}$also.

We have already seen that every almost Archimedean pseudo-lattice $V$ has the property that $V^{+\sim}$ gives a lattice order. The main purpose of this paper is to show that the converse of the previous theorem holds in a 'finite-dimensional' case. Designating the set $\left(x-V^{+}\right) \cap V^{+}$by $[0, x]$ we say that an element $x$ in $V^{+}$is finitely generating if $\operatorname{span}[0, x]$ is finite-dimensional. We let $F$ denote the set of finitely generating elements of $V$.

For our representation result we first need a result on extremal functionals which is of interest in itself.

THEOREM 4. Let $V$ be a directed ordered vector space which is almost Archimedean. If $V$ is a pseudo-lattice or if $V$ has the Riesz decomposition property, then the restriction of any extremal functional defined on the whole space to any order-convex directed subspace is still extremal.

Proof. Let $W$ be an order-convex directed subspace of $V$ and let $f$ be extremal on $V$. If we designate $\left.f\right|_{W}$ by $g$ we will prove that $g$ is extremal on $W$ by showing that Hayes' criterion (Jameson (1970), p. 31) holds. Given $x \in W \cap V^{+}$and $y \in V^{+}$ 
suppose $z \in V$ is such that $\{x, y\} \geqslant z$. If $V$ has the Riesz property, then there exists $q \in V$ such that $\{0, z\} \leqslant q \leqslant\{x, y\}$ and we have $q \in W$, since $0 \leqslant q \leqslant x$ and $W$ is order convex. If $V$ is a pseudo-lattice, let $q=(x+y)-(x \vee y)$ (where $x \vee y$ is taken in the $V^{+\sim}$ order). Then $\{x, y\} \geqslant z$ implies $\{y, x\} \leqslant x+y-z$ which gives $q+\alpha x \geqslant z$ for all $\alpha>0$ by the least property of $x \vee y$. Similarly, $q+\alpha x \geqslant 0$. On the other hand, we have

$$
q-\alpha x=(x+y)-(x \vee y+\alpha x)=\left\{\begin{array}{l}
y-(x \vee y-x+\alpha x) \\
x-(x \vee y-y+\alpha x)
\end{array}\right\} \leqslant\left\{\begin{array}{l}
y \\
x
\end{array}\right.
$$

which shows $q \in W$ since $-\alpha x \leqslant q \leqslant x+\alpha x$ and $W$ is order-convex. In either case we have, for $x \in W \cap V^{+}$and $y \in V^{+}$,

$$
\sup \{f(z):\{x, y\} \geqslant z \in V\}=\sup \{f(q):\{x, y\} \geqslant q \in W\} .
$$

Thus Hayes' criterion for extremality is satisfied for $\{x, y\} \subset W \cap V^{+}$and since $W$ is assumed directed any pair in $W$ can be translated to $W \cap V^{+}$.

THEOREM 5. Let $(V, u)$ be an almost Archimedean order unit space and suppose that $V$ has the Riesz decomposition property or that $V$ is a pseudo-lattice. Then the representation $V \rightarrow \hat{V} \subset \mathbf{R}^{E(V, u)}$ described in Metzler (1975), p. 431, is such that the image of $F-F$ is a selected order space. $(E(V, u)$ is the set of extremal linear functionals which send $u$ to 1 .)

Proof. We will show first that if $x \in F$ and $W=\operatorname{span}[0, x]$ then

$$
\widehat{W}=Z=\left\{g \in \mathbf{R}^{E(V, u)}: \operatorname{spt} g \subset \operatorname{spt} \hat{x}\right\} .
$$

We remark that $\hat{V}^{+}$is composed of nonnegative functions and that $V \rightarrow \hat{V}$ is one-to-one since $V$ is almost Archimedean (Metzler (1975), p. 431).

Let $n=\operatorname{card}(\operatorname{spt} \hat{x})$ and let $\left\{z_{1}, \ldots, z_{k}\right\} \subset[0, x]$ be a basis for $W$. Since this basis maps to a linearly independent subset of $Z$ we see that $k \leqslant n$.

Now if $V$ is a pseudo-lattice or if $V$ has the Riesz decomposition property it is easy to see that the same is true of $W$. Either of these properties implies that the dual of $W$ is a vector lattice and hence (Metzler (1969), p. 6) any set of extremal functionals in the dual of $W$ such that no functional in the set is a multiple of any other one is a linearly independent set. Suppose $\{f, g\} \subset \operatorname{spt} \hat{x}$ and $\left.f\right|_{W}=\left.\alpha g\right|_{W}$ for some $\alpha>0$. Then by $\left({ }^{*}\right)$, established in the proof of the previous theorem, we have, for $r$ sufficiently large,

$$
\begin{aligned}
f(u) & =f(u) \wedge f(r x)=\sup \{f(q):\{u, r x\} \geqslant q \in W\} \\
& =\sup \{x g(q):\{u, r x\} \geqslant q \in W\}=\alpha g(u) \wedge \alpha g(r x)=\alpha g(u) .
\end{aligned}
$$

Since $f(u)=g(u)=1$ we conclude that $\alpha=1$. Now for any $p$ in $V^{+}$and $r$ sufficiently large we have, using $\left(^{*}\right)$ again, $f(p)=f(p) \wedge f(r x)=g(p) \wedge g(r x)=g(p)$. Thus $f=g$ 
on $V^{+}$and we see that $f=g$ on $V$, since $V$ is directed. Then no functional in the restriction of $\operatorname{spt}(\hat{x})$ to $W$ is a multiple of any other and we conclude that $n \leqslant k$ by linear independence. Hence $n$ is equal to $k$.

Now if $y \in V$ is such that $\hat{y}$ is nonnegative and $\operatorname{spt}(\hat{y})=\operatorname{spt}(\hat{x})$ we note first that $\hat{y} \in Z=\hat{W}$. All extremal linear functionals on $W$, by the linear independence argument above, will be multiples of those in $\operatorname{spt}(\hat{x})$. Since these are all positive on $y$ we conclude (Metzler (1975), p. 431) that $y \geqslant \alpha x$ for $\alpha$ sufficiently small, and so $y \in W \cap V^{+}$as required.

As was seen in Metzler (1975), in the case when $V$ has the Riesz decomposition property, we have the additional property that, for $x$ and $y$ in $F, x \wedge y$ (pointwise order) is also a positive element. The converse also holds in any selected order space.

\section{References}

G. Jameson (1970), Ordered linear spaces (Springer-Verlag, Berlin, 1970).

R. Metzler (1969), 'Representation of ordered vector spaces with the Riesz decomposition property', J. London Math. Soc. (2) 1, 3-10.

R. Metzler (1975), 'Representation of ordered linear spaces', Rocky Mountain J. Math. 5, 427-443.

Department of Mathematics

University of New Mexico

Albuquerque, New Mexico 87131

U.S.A. 